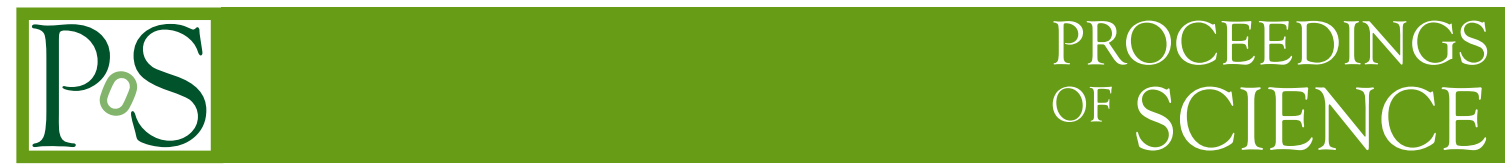

\title{
Flavor physics techniques and sensitivities at $\mathrm{LHCb}$
}

\section{Olivier Deschamps*, on behalf the LHCb collaboration}

Laboratoire de Physique Corpusculaire de Clermont-Ferrand, CNRS/IN2P3/UBP

E-mail: odeschamein2p3.fr

$\mathrm{LHCb}$ is a dedicated experiment aimed at searching for New Physics in the decays of heavy flavors at the LHC. After introducing the detector and trigger, a brief overview of the final state reconstruction is presented. We also discuss the expected physics performance for few key B decay channels.

Flavor Physics and CP Violation 2009,

May 27 - June 1, 2009,

Lake Placid, NY, USA

\footnotetext{
* Speaker.
} 


\section{Introduction}

Within the Standard Model (SM), quark flavor dynamics is described by the KobayashiMaskawa (KM) mechanism which is nicely supported by current B, D and Kaon meson data. The $\mathrm{KM}$ mechanism is now well established as the dominant source of CP violation in the Kaon and $\mathrm{B}$ meson sectors.

Much impressive experimental progress has been made in flavor physics over the past decade and a quite precise determination of the parameters of the Cabibbo-KobayashiMaskawa (CKM) matrix have been achieved from the existing data[2]. Particularly interesting are the parameters, $\bar{\rho}$ and $\bar{\eta}$, which define the apex of the $b-d$ Unitarity triangle. The non-zero value of the latter is the sole source of CP violation in the quark sector. The value of $\bar{\eta}$ is mostly constrained experimentally by the measurements of $\sin (2 \beta)$ and $V_{u b}$, leading to a relative uncertainty of about $5 \%$ in its value. The parameter, $\bar{\rho}$ is dominated by the measurements of the oscillation frequencies $\Delta m_{d}$ and $\Delta m_{s}$, the CKM angle $\alpha$, and $\varepsilon_{K}$, but its value is only known to an accuracy of about $20 \%$. Clearly, the CKM metrology has not entered into the high precision era and still there is room for a sizeable contribution from New Physics from

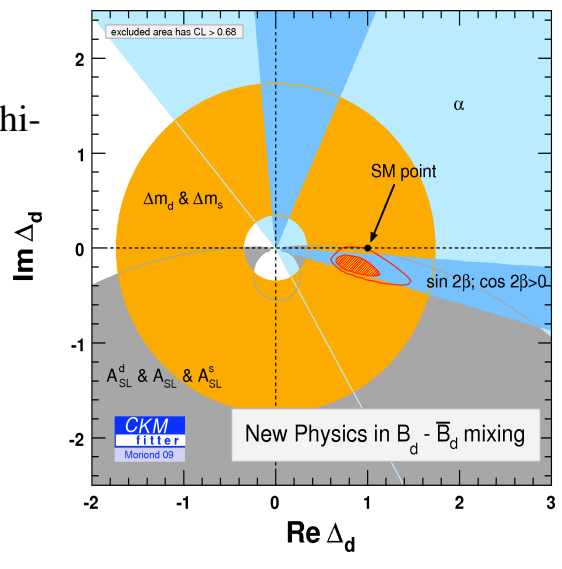

Figure 1: Constraints in the complex plane on the model-independent parameter $\Delta_{d}$ accounting for New Physics in the $B_{d}$ meson mixing. flavour changing processes as illustrated in Fig. 1.

$\mathrm{LHCb}$ is a dedicated heavy flavor physics experiment. Its main goal is to perform high precision measurements in the beauty sector to over constrain the KM scheme and track down the indirect manifestion of New Physics (NP).

\section{The $\mathrm{LHCb}$ detector}

The LHCb detector [1], illustrated in Fig. 2, is a single arm forward spectrometer. It covers an acceptance corresponding to the large pseudo-rapidity region, from 1.9 to 4.9 , where pairs of $b$ quarks are mostly produced in pp collisions. It consists of a precise Vertex Locator (Velo) which is complemented by tracking systems before (TT) and after (T) the Magnet to reconstruct charged particles, two Cherenkov detectors (RICH), which provide an efficient particle identification, the Calorimeter system and the Muon chambers. The main challenge of LHCb is to perform precision measurements in a difficult hadronic environment : the average charged particle multiplicity in the $\mathrm{LHCb}$ acceptance is 30 , and the background from inelastic processes is approximately 200 times larger than bottom production cross-section. Moreover, $\mathrm{LHCb}$ is interested in observing rare or very rare $B$ decays that have branching fractions in the range from $10^{-6}$ to $10^{-9}$, which requires excellent suppression of backgrounds. On the other hand, the rate of b production in LHC collisions is huge and all B species are produced yielding large samples of $B_{s}$ mesons, as well as $B_{c}$ and b-Baryons, which are poorly studied so far. 


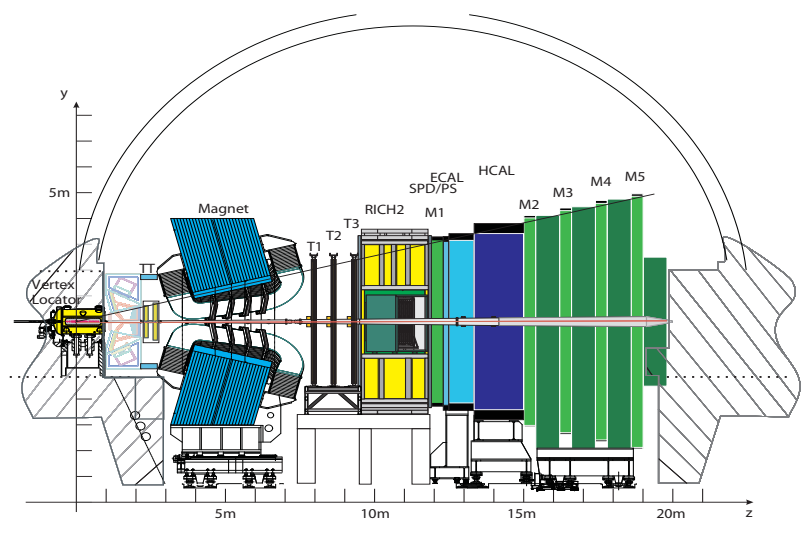

Figure 2: Transversal view of he LHCb detector.

The LHC machine is designed to operate at $14 \mathrm{TeV}$ with a bunch crossing rate of $40 \mathrm{MHz}$. Due to gaps in the bunch structure, the actual rate for the non-empty bunch crossings is $30 \mathrm{MHz}$. At the LHCb crossing region, the beam focusing is optimized to obtain a luminosity at the level of a few times $10^{32} \mathrm{~cm}^{-2} \mathrm{~s}^{-1}$ in order to maximise single pp-interactions bunch crossings. The expected nominal integrated luminosity per year is $2 \mathrm{fb}^{-1}$. During the start-up stage in late 2009, the LHC will operate in a different conditions; lower energies of 7 to $10 \mathrm{TeV}$ and much smaller number of bunches. A few hundred $p b^{-1}$ of data are expected to be collected. Within the first day(s), LHCb should collect $10^{8}$ minibias events with simple trigger criteria. To move toward the $B$ meson study, a specific B-trigger is to be setup as discussed in the next section.

\section{LHCb trigger strategy}

The LHCb triggering is performed in two steps. The first trigger level (L0) is fully hardware and aims at reducing the data rate to $1 \mathrm{MHz}$.

The L0 trigger exploits the higher transverse momentum of particles from B decay to provide about a factor of 10 reduction in the minimum bias rate with only about a $50 \%$ loss in efficiency. The L0 is based on the the fast response of the calorimeters and the muon detectors to identify high transverse momentum hadrons, electrons, photons, neutral pions and muons that could

\begin{tabular}{|c||c|c|c|}
\hline decay channel & Level-0 & HLT1 & HLT2 \\
\hline \hline Muonic & $90 \%$ & & \\
Electromagnetic & $70 \%$ & $\sim 80 \%$ & $>90 \%$ \\
Hadronic & $50 \%$ & & \\
\hline
\end{tabular}

Table 1: Efficiency of the successive levels of the $\mathrm{LHCb}$ trigger on selected events for decay channels containing muons, electrons or photons, and hadronic final states, respectively. signal the presence of a B decay. The typical thresholds on transverse momentum is $1 \mathrm{GeV} / \mathrm{c}$ for muons and 3-4 GeV/c for the deposition in the hadronic and electromagnetic calorimeters. The typical bandwidth sharing is $7: 2: 1$ for hadronic, electromagnetic and muon trigger lines, respectively, leading to a $90 \%$ efficiency for B decay channels containing muons, $70 \%$ for those containing electrons or photons and $50 \%$ for decays with hadronic final states, which can be reconstructed offline. 
The second level (High Level Trigger) is purely software. In the first step (HLT1), the high transverse momentum objects found in $\mathrm{L} 0$ are confirmed using additional information from the tracking and the Velo systems, and additional impact parameter can be applied. The $1 \mathrm{MHz}$ input rate into HLT1 is reduced to about $40 \mathrm{kHz}$, which is then processed by a second software level, HLT2. HLT2 performs a global event reconstruction using the full information from the LHCb detector. The performance of the L0 and HLT trigger levels are summarized in the Table 1 for decay channels containing muons, electrons or photons, and hadronic final states, respectively.

After these two levels of triggering, the events are written to permanent storage at a rate of $2 \mathrm{kHz}$ with an average event size of $35 \mathrm{kB}$.

\section{Resolving the event topology}

\subsection{Reconstructing the final state kinematics}

The LHCb tracking system is $95 \%$ efficient for reconstructing a charged particle with a momentum above $10 \mathrm{GeV} / \mathrm{c}$ and achieves a momentum resolution of about $0.5 \%$.

The typical mass resolution for B decay into a charged particles final state is around $15 \mathrm{MeV} / \mathrm{c}^{2}$ as illustrated in Fig. 3 for the $B_{s} \rightarrow D_{s}(K K \pi) K$ decay. The good mass resolution allows for an efficient suppression of the remaining background from $B_{s} \rightarrow D_{s} \pi$ decay with the bachelor pion misidenitfied as a kaon.

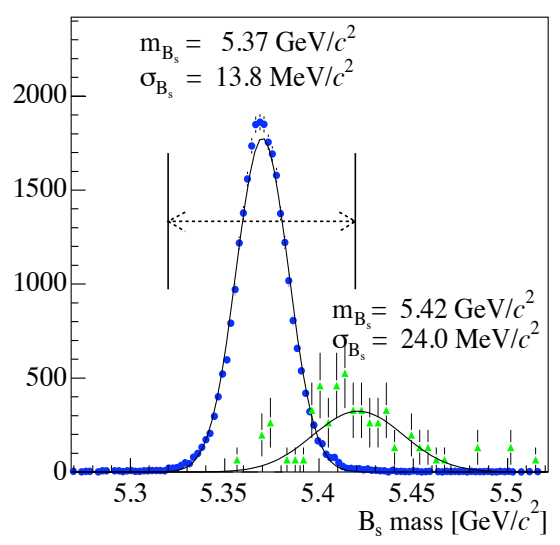

Figure 3: Reconstructed mass for the $B_{s} \rightarrow D_{s}(K K \pi) K$ decay. The shifted distribution from remaining $B_{s} \rightarrow D_{s} \pi$ background is shown.

A large sample of long-living strange particle will be produced at LHCb. With the first data taking run, a clear signal from $K_{s} \rightarrow \pi \pi$ and $\Lambda \rightarrow p \pi$ is expected. Applying simple kinematical and vertex cuts only, a sample with a purity of $95 \%$ can be selected and will be used to obtained cleanly identified protons and pions for the calibration of the RICH particle identification. $K_{s}$ and $\Lambda$ also appears in the final state of several decays of interest for LHCb physics program, such as $B \rightarrow J / \Psi K_{s}, D \rightarrow K_{s} \pi \pi$ and $\Lambda_{b}$ decays. The reconstruction efficiency for these long-lived particle decaying in the LHCb acceptance is $60 \%$ with $2 / 3$ of them decayed beyond the vertex detector.

The reconstruction efficiency for photons that are in calorimeter acceptance with a transverse momentum above 200 $\mathrm{MeV} / \mathrm{c}$ is around $70 \%$. Radiative B decays such as $B_{s} \rightarrow$ $\Phi \gamma$ and $B_{d} \rightarrow K^{* 0} \gamma$ can be reconstructed with a mass resolution of $90 \mathrm{MeV} / c^{2}$, mostly dominated by the electromagnetic calorimeter (Ecal) energy resolution.

Low energy $\pi^{0}$ 's can be reconstructed as a pair of well separated photons with a mass resolution of $10 \mathrm{MeV} / c^{2}$. For $\pi^{0}$ 's with transverse energy above $2 \mathrm{GeV}$, the distance between the two photons becomes of the order of the Ecal granularity, and the photons are likely to be merged into a single Ecal cluster. A dedicated reconstruction procedure has been developed to reconstruct the overlapping photons pair from the cluster's transverse shape. A mass resolution of about $20 \mathrm{MeV} / c^{2}$ is achieved 
for this high energy $\pi^{0}$ which is important for reconstruction of such decays as $B \rightarrow \pi^{+} \pi^{-} \pi^{0}$ decay. The overall reconstruction efficiency is $50 \%$ up to a transverse energy close to $10 \mathrm{GeV}$ for neutral pions that decay in the $\mathrm{LHCb}$ acceptance.

\subsection{The particle identification}

Efficient hadron identification over a wide range of momentum is achieved using two RICH detectors with three different radiator materials.

The pair of RICH's provide efficient $\pi-K$ separation up to a momentum of $100 \mathrm{GeV} / \mathrm{c}$, with a Kaon identification efficiency of $97 \%$ for a misidentification probability of $6 \%$. The impact of the RICH identification on $B \rightarrow h^{+} h^{-}$decay separation is shown Figure 4.

The lepton identification is accomplished by the use of the calorimeter and muon systems. Efficient electron identification is provided by the calorimeters information based on the matching of tracks reconstructed by the tracking system and Ecal clusters, the energy deposit in the first electromagnetic sampling layer (Preshower) and the reconstruction of the BremStrahlung photon associated with the charged track. A $3 \%$ misidentification rate is expected for a $95 \%$ electron identification efficiency. Good electron ID is needed for the flavor tagging of semi-leptonic B decay and gives access to several B decay modes, such as $B \rightarrow K^{*} e^{+} e^{-}$or $B \rightarrow J / \Psi\left(e^{+} e^{-}\right) K_{s}$.
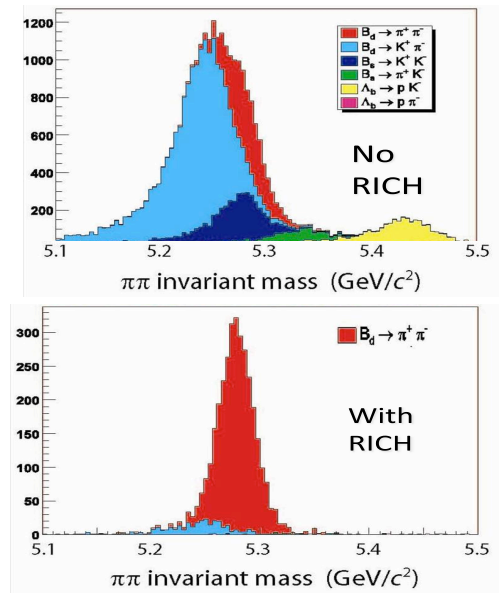

Figure 4: Reconstructed mass for the $B_{s} \rightarrow \pi^{+} \pi^{-}$decays without RICH identification (top) and using RICH identification (bottom) . The muon identification is dominated by the information from the muon chambers which provides a $1 \%$ misidentification rate for a $95 \%$ identification efficiency.

\subsection{The proper time measurement}

The measurement of the proper time of the decaying B is a key ingredient in the many LHCb's core analyses. The LHCb Vertex Locator is a silicon strip detector with the first measurement at a radius of $8.2 \mathrm{~mm}$ from the beam and has a hit resolution of better than $10 \mu \mathrm{m}$. It provides a precise impact parameter determination with an expected resolution of $14 \mu m+35 / p_{T}\left(p_{T}\right.$ in $\left.\mathrm{GeV} / \mathrm{c}\right)$ which allows for efficient rejection of combinatorial background arising from particles coming from the primary vertex. The primary vertex position is reconstructed with a $50 \mu \mathrm{m}$ resolution along the beam axis (z-axis) and 5-10 $\mu \mathrm{m}$ transverse to the beam direction. Displaced vertices are reconstructed with a precision along the z-axis of about $150 \mu \mathrm{m}$ as compared to the $1 \mathrm{~cm}$ average flight distance for $\mathrm{B}$ mesons in LHCb. When the position uncertainty is combined with the precision of the momentum measurement, it results in a decay time resolution of about $40 \mathrm{fs}$ for $B$ mesons decaying into charged particles only, and about $80 \mathrm{fs}$ for radiative $B$ decay or $B$ decays with an energetic $\pi^{0}$.

This excellent proper time resolution is critical to resolve the fast oscillations of $B_{s}$ mesons, which is a key ingredient to many of the time-dependent CP measurements in $B_{s}$ decays. This time resolution can be accurately extracted from the analysis of $B_{s} \rightarrow D_{s} \pi$ decays $\left(170 \times 10^{3}\right.$ selected event expected each year), which would then be directly applicable to $B_{s} \rightarrow D_{s} K$, for example. 


\subsection{Flavor tagging}

In addition to excellent proper time resolution, $\mathrm{CP}$ asymmetries also rely on tagging the flavor of the B meson at production (so called, flavor tagging). LHCb applies the classical flavor tagging techniques, combining the information from the opposite-side $\mathrm{b}$ (kaon-tag from $b \rightarrow c \rightarrow s$ cascade, lepton-tag for semi-leptonic $B$ decay and global vertex-charge) and the fragmentation information from the signal side $\mathrm{b}$. Unlike the B-factories operating at the $\Upsilon(4 S)$, the tagging $B$ produced in hadronic collisions oscillate incoherently : almost $50 \%$ of the tagging-b form a neutral $B$ meson, including $10 \%$ to the rapidly oscillating $B_{s}$, and thus contribute to the dilution of the b-tagging power. LHCb's RICH detectors provides excellent kaon-tagging for both the opposite-side (both $B$ and $B_{s}$ ) and the same-side tagging in case of a $B_{s}$. As an illustration, the tagging efficiency for the $B_{s} \rightarrow J / \Psi \Phi$ decay is $55 \%$ with a wrong tagging rate of $33 \%$, resulting in a tagging power of $6.2 \%$, half of which comes from the kaon-tag information. The typical tagging power for $B_{d}$ decay is slightly lower, at the level of $5 \%$, due to the reduced power of the same-side tagging with pions.

The extraction of the tagging performance from the data is likely to come first for the oppositeside taggers, as there are higher statistics for $B_{u}$ and $B_{d}$ channels with respect to $B_{s}$. From the self tagging $B_{d} \rightarrow J / \Psi K^{*}$ decay, the wrong tagging rate canb be extracted from the data with a $0.3 \%$ statistical uncertainty with one nominal year of data taking.

\section{Probing flavor physics}

\subsection{Measuring a branching ratio of a very rare decay $: B_{s} \rightarrow \mu^{+} \mu^{-}$}

The $B_{s} \rightarrow \mu^{+} \mu^{-}$decay is a FCNC transition dominated by the Z-penguin diagram. It's rate is well predicted by the SM [2] and is expected to have a branching ratio of $\left(3.291_{-0.267}^{+0.094}\right) 10^{-9}$.

New Physics particle entering into the loop can modify significantly this branching ratio. This decay is thus an efficient probe for New Physics. The LHCb selection for $B_{s} \rightarrow \mu^{+} \mu^{-}$is based on a 3-dimensional likelihood [3]. The first likelihood dimension combines the various geometrical information on the decay (impact parameter, $B_{S}$ decay length, vertex isolation, etc) and is illustrated in Figure 5. The other two dimensions rely on the reconstructed $B_{s}$ mass and the muon-identification, respectively. The likelihood distributions are fully determined from the data in order to limit the Monte-Carlo dependence of the analysis. Reconstructed mass side-bands are used to determine the background distributions and the signal distributions will be evaluated using control channels including

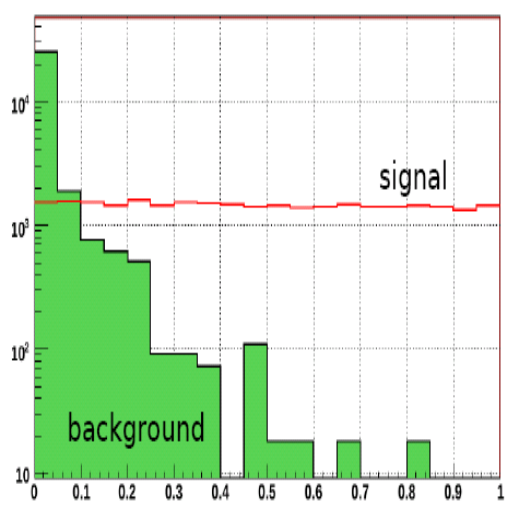

Figure 5: Geometrical Likelihood distribution for $\mu^{+} \mu^{-}$signal and background. $B_{(s)} \rightarrow h^{+} h^{-}$or $B_{d} \rightarrow J / \Psi(\mu \mu) K_{s}$.

Channels with known branching fractions will be used to derive the absolute branching fraction from the yield of selected events. The trigger efficiency and tracking efficiencies are normalized using $B_{d} \rightarrow K \pi$ and $B_{u} \rightarrow J / \Psi(\mu \mu) K^{-}$. The main systematics (13\%) come from the ratio of hadronization rates of the $B_{s}$ relative to the $B_{d}$ and $B_{u}$ mesons $\left(f_{B_{s}} / f_{B_{d, u}}\right)$. The likelihood distribu- 
tion are analyzed on a statistical basis to extract the results shown in Figure 6. With an accumulated luminosity as small as $0.1 \mathrm{fb}^{-1}$, LHCb is expected to reach the final combined limit from the Tevatron [4]. A $3 \sigma$ evidence of the Standard Model branching ratio could show up with one nominal year of data taking and a $5 \sigma$ observation in several years.
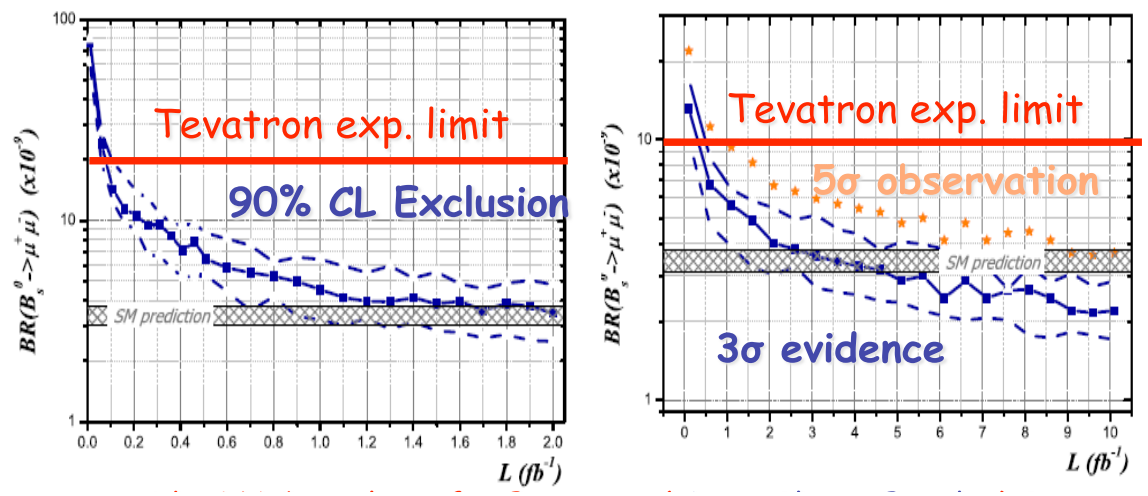

Figure 6: $90 \% \mathrm{CL}$ upper limit on the $B_{s} \rightarrow \mu^{+} \mu^{-}$branching ratio (left) and $3 \sigma$ evidence and $5 \sigma$ observation (right) as a function of the integrated luminosity. The expected Tevatron performance is shown.

\subsection{Measuring a time-dependent decay rate : the photon polarisation in $B_{s} \rightarrow \Phi \gamma$}

Another probe of New Physics is the measurement of the photon polarization in the radiative $b \rightarrow q \gamma$ penguin transition. In the Standard Model the photon from $b \rightarrow s \gamma$ is mostly left-handed. The right-handed component is suppressed by the ratio of the strange quark mass to the b-quark mass. The time-dependent decay rate of the radiative decay for initial $B_{q}, \bar{B}_{q} \rightarrow f^{C P} \gamma$, where $f_{C P}$ is a CP eigenstate, exhibits a term, $A_{q}^{\Delta}$, dependent on the fraction of wrong photon polarization.

$\Gamma\left(B_{q}\left(\bar{B}_{q}\right) \rightarrow f^{C P} \gamma\right)=\|A\|^{2} e^{-\Gamma_{q} \tau}\left[\cosh \left(\Delta_{q} \tau / 2\right)+A_{q}^{\Delta} \sinh \left(\Delta_{q} \tau / 2\right) \pm C_{q} \cos \left(\Delta m_{q} \tau\right) \mp S_{q} \sin \left(\Delta m_{q} \tau\right)\right]$

Within the Standard Model, and in the case of the $B_{s}$ meson one expects the time dependent and time-independent asymmetry terms, $S_{s}$ and $C_{s}$, almost to vanish. The large lifetime difference for $B_{s}$ ensures some sensitivity to the polarization amplitude ratio, $A_{q}^{\Delta}$. The reliable SM prediction [6] at the next-to-next leading order for this polarization term can be confronted with the experimental data to check for New Physics in loop.

Approximately $11 \times 10^{3} B_{s} \rightarrow \Phi \gamma$ signal events are expected to be recosntructed and selected with LHCb per nominal year of data taking with a background over signal ratio of less than $90 \%$ [5]. From the flavour-tagged time-dependent decay rate, the polarization amplitude and the asymmetry parameters can be simultaneously obtained. The background is parametrized from the reconstructed mass side-bands and the proper-time acceptance from the control channels $B_{d} \rightarrow K^{*} \gamma$ and $B_{s} \rightarrow J / \Psi \Phi$. A resolution of 0.22 is expected on the polarization amplitude and 0.1 for the asymmetry parameters, $C_{s}$ and $S_{s}$ with one nominal year of data taking.

\subsection{A Dalitz plot analysis : the UT angle $\gamma$ from $B^{-} \rightarrow D^{0}\left(K_{s} \pi^{+} \pi^{-}\right) K^{-}$}

An important goal of the $\mathrm{LHCb}$ experiment is to precisely determine the Unitarity Triangle angle $\gamma$. This phase can be measured, for instance, through the interference between the $b \rightarrow c$ and 
$b \rightarrow u$ transitions in $B^{-} \rightarrow D K^{-}$.

Among several methods, a possible technique to extract simultaneously the weak phase $\gamma$, the strong phases and the decay amplitudes ratio, is to perform a Dalitz plot analysis of the $D^{0}$ and $\bar{D}^{0} 3$-body decay into $K_{s} \pi^{+} \pi^{-}$.

With the nominal annual luminosity of $2 \mathrm{fb}^{-1}, 5 \times$ $10^{3}$ signal events are expected to be selected in $\mathrm{LHCb}$ with a background over signal ratio less than 70\% [7]. The angle $\gamma$ can be extracted using an unbinned amplitude fit, assuming an isobar model for the various resonances entering in the Dalitz plot. With this method a statistical resolution at the level of 10 degrees can be achieved on $\gamma$ together with a 7 degrees systematics uncertainty due to the modeling of the intermediate resonances. To limit the model dependence, a binned fit using inputs on the strong phase shifts from the correlated $\Psi \rightarrow D^{0} \bar{D}^{0}$ decay at CLEO-c can be done. It results in a significant reduction

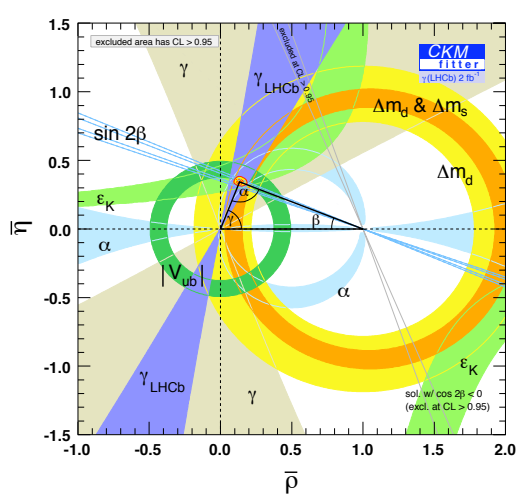

Figure 7: Constraints in the $(\bar{\rho}, \bar{\eta})$ plane from the global CKM fit of the current data. The expected constraint from $\gamma$ with 1 nominal year of LHCb data taking is superimposed. in the systematics uncertainty, at the level of 1-2 degrees, and thus will be of great importance when the statistical precision approaches 10 degrees. This Dalitz method can be extended to other $D^{0}$ decays, such as $K_{s} K K$ or $K_{s} K \pi$. In addition, there are several other methods to extract the $\gamma$ angle from tree processes [7]. Combining all these techniques the LHCb resolution on $\gamma$ is expected to reach the 5 degrees level with an integrated luminosity of $2 \mathrm{fb}^{-1}$. The impact of such a measurement on the global CKM fit is illustrated in Figure 7.

\subsection{The forward-backward asymmetry from the angular analysis of the $B_{d} \rightarrow K * \mu \mu$ decay.}

The $B_{d} \rightarrow K^{*} \mu^{+} \mu^{-}$decay proceeds via the suppressed FCNC $b \rightarrow s$ Electroweak-penguin transition with a measured branching fraction $\left(1.03_{-0.23}^{+0.26}\right) 10^{-6}[8]$ in good agreement with the SM expectation. The dynamics of this decay are very sensitive to any New Physics particles that possibly enters into the flavor-changing loop. In particular the lepton angular distribution in the dilepton rest-frame with respect to the B momentum is expected to be significantly modified by many New Physics scenarios. There are severable observables to test the decay dynamics, including the muon forward-backward asymmetry as a function of the di-muon invariant mass squared. The zero-crossing point of this asymmetry could discriminate between several New Phyics models as illustrated in the left part of Figure 8.

The Standard Model prediction for the zero-crossing point of this asymmetry depends on the Wilson Coefficients, $C_{7}$ and $C_{9}$ and is predicted to be $m_{\mu \mu}^{2}(0)=\left(4.36_{-0.31}^{+0.33}\right) \mathrm{GeV}^{2} / \mathrm{c}^{4}$ [9]. About $7 \times 10^{3}$ signal events are expected to be selected per nominal year with a background over signal ratio of less than $20 \%$ in LHCb [10]. The expected resolution on the zero-crossing point is $0.5 \mathrm{GeV}^{2} / c^{4}$. A full angular analysis of the decay with high statistics is expected to provide a better discrimination between the New Physics models. 

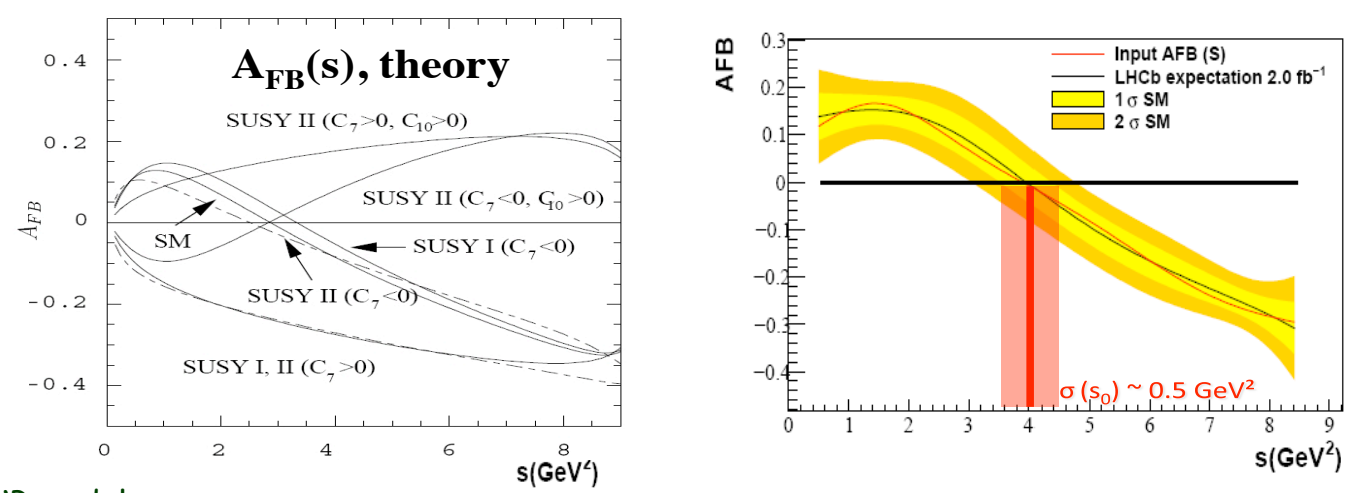

Figure 8: Muon forward-backward asymmetry in the $B_{d} \rightarrow K^{*} \mu^{+} \mu^{-}$decay as a function of the di-muon invariant mass for the Standard Model and various SUSY models (left) and the expected LHCb measurement performance (right).

\subsection{A time-dependent flavor-tagged angular analysis : the UT angle $\beta_{s}$ from $B_{s} \rightarrow J / \Psi \Phi$}

The $B_{s} \rightarrow J / \Psi \Phi$ decay data allow for the extraction the $B_{s}$ mixing phase, $\phi_{s}$, with respect to the $b \rightarrow c$ decay phase, from an almost pure $b \rightarrow c c s$ tree transition, and thus is sensitive to New Physics in the $B_{s}$ box diagram.

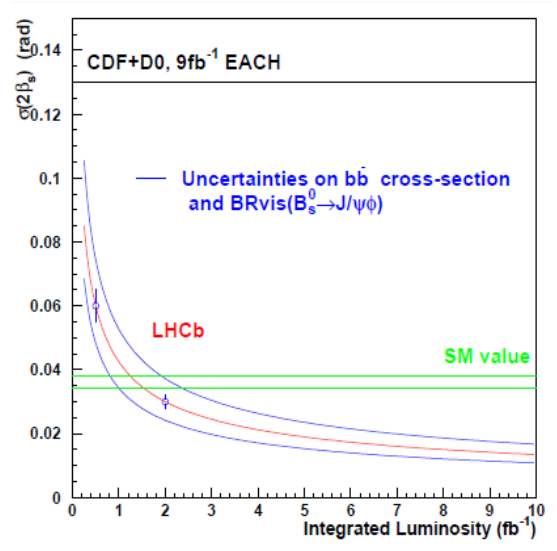

Figure 9: $\mathrm{LHCb}$ expected resolution on the mixing phase $2 \beta_{s}$ from $B_{s} \rightarrow J / \Psi \Phi$ as a function of the luminosity.
The expected small value of the $B_{s}$ mixing phase is well determined within the Standard Model by the CKM metrology : $\phi_{s}=-2 \beta_{s}=0.0362 \pm 0.0018$ [2]. The large central value recently observed in the direct measurement by the CDF and D0 experiments,

$\Phi_{J / \Psi \Phi}=0.76 \pm 0.36[11]$ deviates by more than $2 \sigma$ with respect to the Standard Model expectation. This decay is thus an important channel to be analyzed by LHCb [12].

As in any vector-vector final state in a $B$ decay, the $J / \Psi \Phi$ is not a pure CP-eigenstates. An angular analysis is needed to separate on a statistical basis the CP-odd from the CP-even components. A large yield of about 120,000 signal events is expected to be collected per year with a background over signal ratio of about 2 . An unbinned likelihood fit to the time dependent decay rates for the initial $B_{S}$ and $\bar{B}_{S}$ allows one to extract the mixing phase $\phi_{s}$ together with the lifetime and the even and odd components' parameters. A resolution on $\phi_{s}$ of 0.03 radians can be expected with $2 f b^{-1}$. The expected resolution on $\phi_{S}$ as a function of the integrated luminosity is displayed in Figure 9. This analysis requires an accurate control of the acceptance, of the flavor tagging and of the background level. This is to be done using the control channels $B \rightarrow J / \Psi K^{*}, B^{+} \rightarrow J / \Psi K^{+}$and $B_{s} \rightarrow D_{s}^{*} \pi$, and the selected signal mass side-bands.

A similar analysis can be done with the pure $b \rightarrow$ sss gluonic penguin transition, $B_{s} \rightarrow \Phi \Phi$. The dependency on $V_{t s}$ in both the mixing and the decay amplitudes leads to a cancellation of the 
$B_{s}$ mixing phase in this decay. Therefore, if any significant phase is measured, this would be a clear signature of New Physics. Almost $4 \times 10^{3}$ events are to be selected each year leading to a statisical resolution of 0.1 on the phase $\phi_{\Phi \Phi}$.

\section{Conclusions}

The LHCb detector is a dedicated experiment to search for New Physics in the decay of heavy flavors. It aims at performing high precision measurements in the challenging environment of the hadronic collisions of the LHC. As briefly outlined in this document, sophisticated techniques for the reconstruction of the final state and for their analysis are in place. Important results, such as the improvement on the limit on the $B_{s} \rightarrow \mu^{+} \mu^{-}$branching fraction and on the measurement of the $B_{s}$ mixing phase are expected to come soon after the LHC startup. The physics program of LHCb covers a much wider range than the few key analysis discussed in Section 5 of this proceeding.

With $\mathrm{LHCb}$, we hope to make a great step forward in our understanding of the dynamics of the heavy flavor decays and uncover an indirect manifestation of physics beyond the Standard Model.

\section{Acknowledgements}

My warmest thanks go to my collaborators of the LHCb collaboration for their support in the preparation of this talk. I also would like to thank the organizers of the FPCP09 conference for their kind hospitality in Lake Placid, NY.

\section{References}

[1] LHCb Collaboration, "The LHCb Detector at the LHC", Journal of Instrumentation, 2008 JINST 3 S08005;

[2] Valentin Niess., "CKM fits", this proceedings; The CKMfitter group, http://ckmfitter.in2p3.fr/;

[3] D. S. Santos et al., "Analysis of the decay $B_{s}^{0} \rightarrow \mu^{+} \mu^{-}$at LHCb", LHCB-ROADMAP1, in preparation

[4] T. Aaltonen et al. [CDF collaboration], Phys. Rev. Lett. 100 (2008) 101802; D0 collaboration, D0 note 5344-CONF (2007);

[5] S. Barsuk et al., "Roadmap for the radiative decays of beauty hadrons at LHCb", LHCB-ROADMAP4, in preparation;

[6] D. Atwood, M. Gronau and A. Soni, Phys. Rev. Lett. 79 (1997) 185;

[7] M. Adinolfi et al., "The tree-level determination of gamma at LHCb ", LHCB-ROADMAP5, in preparation;

[8] C. Amsler et al., Physics Letters B667, 1 (2008);

[9] M. Beneke et al., "Exclusive radiative and electroweak Bd and Bs penguin decays at NLO", Eur.Phys.J.C41:173-188,2005;

[10] J. Dickens et al.,"Roadmap for the analysis of $B_{d}^{0} \rightarrow K^{* 0} \mu^{+} \mu^{-}$at LHCb", LHCB-ROADMAP2, in preparation;

[11] The CDF Collaboration (T. Aaltonen, et al.),Phys. Rev. Lett. 100, 161802 (2008); The D0 Collaboration (V.M. Abazov, et al.), arXiv:0802.2255 [hep-ex] (2008);

[12] J. Albrecht et al.,"Roadmap for the measurement of mixing induced CP violation in $B_{S}^{0} \rightarrow J / \Psi \Phi$ at LHCb", LHCB-ROADMAP3-001, in preparation; 